\section{Active zone self-similarity of fractal- Sierpinski antenna verified using infra-red thermograms}

J.M. González, M. Navarro, C. Puente, J. Romeu and A. Aguasca

The surface current distribution of a Sierpinski fractal antenna shows a self-similar behaviour determined by the self-similar properties of its geometry. The application of infra-red thermography to electromagnetic near field detection allows the experimental verification of the active region scaling of a fractal antenna operating at different bands.

Introduction: The multiband behaviour of the fractal-Sierpinski antenna has been empirically described in terms of its input impedance and radiation patterns at several frequency bands $[1,2]$. The surface current distribution on the antenna has been numerically evaluated by applying the FDTD algorithm [3], and shows a selfsimilar behaviour and scaling of the active region at each operating band. Return losses obtained from this algorithm are in good agreement with the experimental results previously mentioned. In this Letter, it is explained how an infra-red measurement technique can be used to experimentally verify the scaling properties of the active region in a fractal-Sierpinski antenna.

Antenna description: The analysed fractal Sierpinski antenna is constructed by the subtraction of a central inverted equilateral triangle from a main equilateral triangle shape, generating three triangles, each one of the three triangles being half of the size of the original one. By iterating an infinite number of times the same subtraction procedure on the remaining triangles, the ideal Sierpinski gasket is obtained. At each iteration new triangular clusters appear in the structure. These clusters are scaled by a factor of 2 at each iteration. The height of these triangular clusters is $h / 2^{n}$, where $h$ is the total height of the antenna and $n=0,1, \ldots, 4$.

The antenna self-similarity gives rise to a scalable surface current distribution. This means that the current distribution on the Sierpinski antenna is scaled at each band of operation. The area with a high current density concentrates towards the vertex of the feeding point of the antenna in the same proportion as the operating wavelength. The scalability of the active region together with the existence of the same characteristic parameters (input matching and radiation pattern) at several bands, also related with a factor of 2, explain the multiband behaviour of this structure. Active regions of a five iteration Sierpinski antenna and surface current distribution obtained with an FDTD algorithm are presented in Fig. 1 for the second, third and fourth bands.

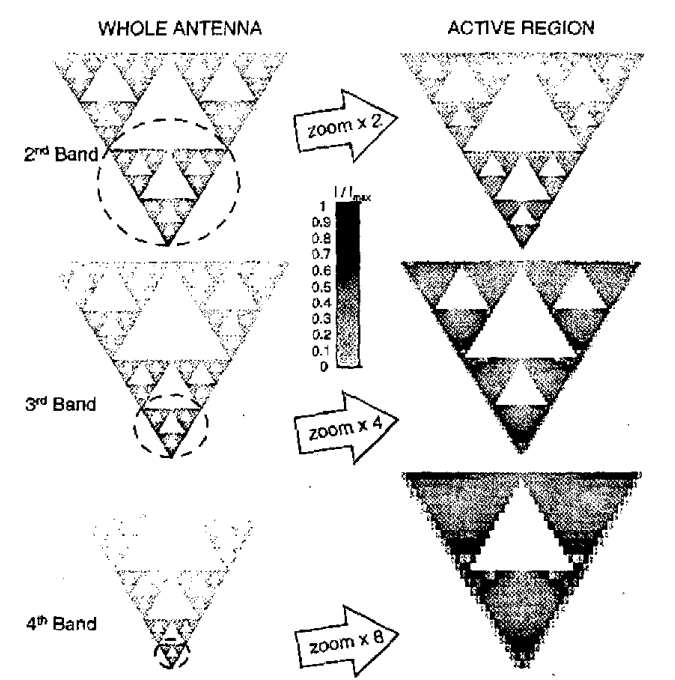

Fig. 1 Surface current distribution on Sierpinski antenna (FDTD simulation) for second, third and fourth bands

This Figure is shown in colour on Electronics Letters Online.
Measurements: To verify the active region scalability predicted by the numerical simulations, an electromagnetic-field measurementtechnique based on infra-red thermograms was used. Infra-red thermography is a technique based on the measurement of the transformation into heat of energy absorbed by a lossy material when an electromagnetic wave impinges on it [4].

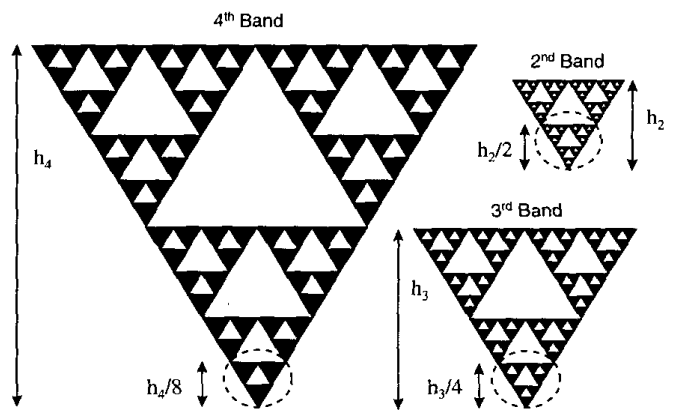

Fig. 2 Fractal Sierpinsiki antennas of five iterations and $60^{\circ}$ tuned at same frequency but at different operating bands (second, third and fourth bands)

Active region dimensions are shown enclosed by a circle
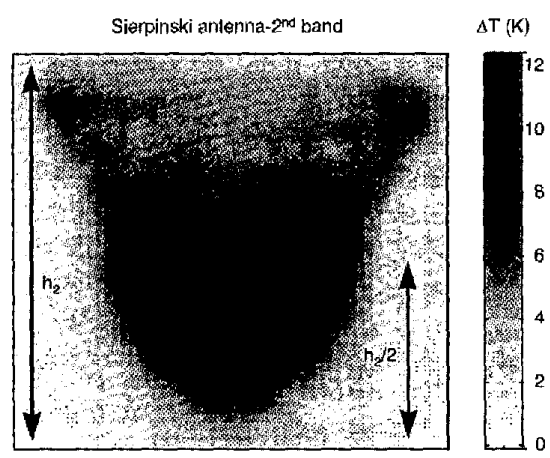

Fig. 3 Infra-red thermogram of second band antenna

Side bar shows temperature increment over ambient temperature. This Figure is shown in colour on Electronics Letters Online.

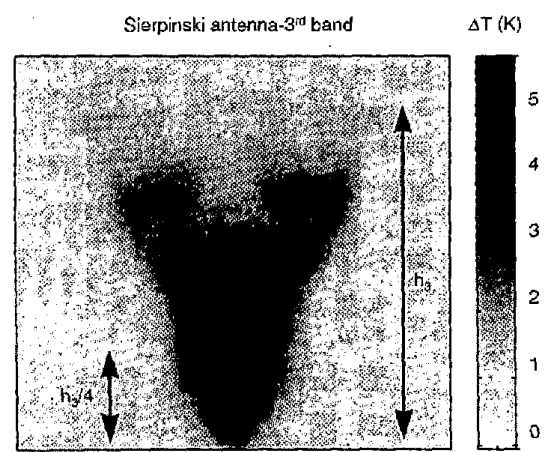

Fig. 4 Infra-red thermogram of third band antenna

This Figure is shown in colour on Electronics Letters Online.

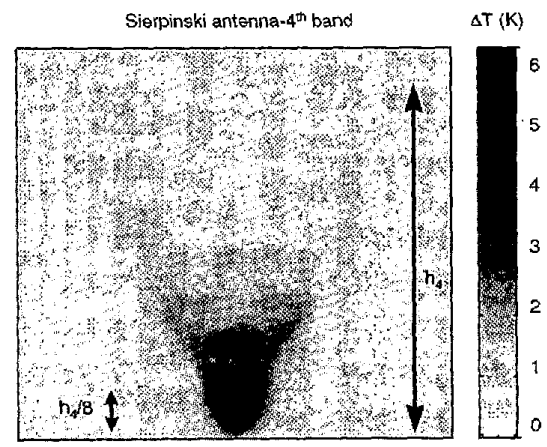

Fig. 5 Infra-red thermogram of fourth band antenna

This Figure is shown in colour on Electronics Letters Online. 
Three Sierpinski antennas were designed and printed on a fibreglass substrate, the electric losses of which enabled the thermal images to be acquired. These three antennas were operated at the same frequency of $2.45 \mathrm{GHz}$, although for each one this frequency was assigned to a different band. The heights of the designed antennas were: $6 \mathrm{~cm}\left(h_{2}\right)$ for the second band antenna, $12 \mathrm{~cm}\left(h_{3}\right)$ for the third band antenna and $22 \mathrm{~cm}\left(h_{4}\right)$ for the fourth band antenna. The scaling factor of 2 for the fourth band antenna was not maintained due to the substrate, which slightly changes the resonant frequency of the antenna. Fig. 2 shows the antenna heights. The antenna with its first band at $2.45 \mathrm{GHz}$ was not measured due to the lack of an appropriate optical lens for the infrared camera in order to carry out the measurement, while the fifth band antenna was not constructed because of its large size. Figs. 3 - 5 show, respectively, the thermograms acquired for the second, third and fourth band antennas when the incident power on them was almost $4 \mathrm{~W}$. As had been previously assumed, by selecting an antenna with a higher operating band we obtain an illuminated area in the thermal images (the area with higher temperatures): $h_{2}$ / 2 for the second band, $h_{3} / 4$ for the third and $h_{4} / 8$ for the fourth. These regions correspond to the active area and coincide with the regions in which the temperatures reached by the fibreglass are higher.

Conclusion: Infra-red thermograms allow experimental verification of the self-similar behaviour of the active region in a Sierpinski antenna, which confirms its multiband characteristics. Active area dimensions are in good agreement with the predictions obtained by means of numeric methods.

Acknowledgment: This work has been supported by grants TIC-960724-C06-04 and FEDER 2FD97-135.

(C) IEE 1999

Electronics Letters Online No: 19990943

DOI: 10.1049/el:19990943

J.M. González (Signal Theory and Communications Area, Centro Politécnico Superior, Universidad de Zaragoza (UZ), C/Maria de Luna n. 3, 50015 Zaragoza, Spain)

E-mail: arbesu@posta.unizar.es

M. Navarro, C. Puente, J. Romeu and A. Aguasca (Electromagnetics \& Photonics Engineering Group, Dept. of Signal Theory and Communications, Universitat Politécnica de Catalunya (UPC),- c/Gran Capità s/n, Mòdul D3 Campus Nord UPC, 08034 Barcelona, Spain)

\section{References}

I Puente, C., Romed, J, POUS, R., GarCia, X., and Benitez, F.: 'Fractal multiband antenna based on Sierpinski gasket', Electron. Lett., 1996, 32, (1), pp. 1-2

2 puente-baliarda, C., Romeu, J., pous, R., and Cardama, a.: 'On the behavior of the Sierpinski multiband fractal antenna', IEEE Trans. Antennas Propag., 1998, AP-46, pp. 517-524

3 PUENTE, C.: 'Fractal antennas'. PhD Dissertation, Universitat Politècnica de Catalunya, Barcelona, June 1997

4 NORGARD, J.D.: 'Infrared/microwave correlation measurements', Opt. Eng., 1994, 33, pp. 85-96

\section{Aperture coupled microstrip patch antenna with thick ground plane in millimetre waves}

\author{
O. Lafond, M. Himdi and J.P. Daniel
}

The finite thickness of the ground plane is taken into account in the cavity model to analyse some aperture coupled microstrip patch antennas at millimetre-wave frequencies $(60 \mathrm{GHz})$. The thickness has a strong effect on impedance matching at high frequencies owing to the ratio between the thickness and the wavelength, which increases with frequency. The calculated results are compared to those obtained by experiment for several antennas with different input impedances due to different slot lengths. Close agreement is found between the calculated and experimental results.
Introduction: In a previous analysis of aperture coupled microstrip patch antennas [1], the antenna represented in Fig. 1 was modelled using the cavity method. At low frequencies, the ground plane thickness $(t)$ is very small compared to the wavelength, and we can therefore consider this thickness to be zero. However, this is not the case at millimetre-wave frequencies $(60 \mathrm{GHz})$ where the classical copper layers are $17.5 \mu \mathrm{m}$ thick, and $35 \mu \mathrm{m}$ if there are two layers. In this Letter, the effect of ground plane thickness is taken into account by introducing an empirical coefficient [2] to improve the model in $\mathrm{V}$ band and to determine the effect on input impedance [3].

Moreover, a thick ground plane may be used to improve the rigidity of thin substrates $(127 \mu \mathrm{m}$ at $60 \mathrm{GHz})$. To verify the accuracy of the model, several aperture coupled patch antennas have been realised and experimental results have been compared to calculated results.
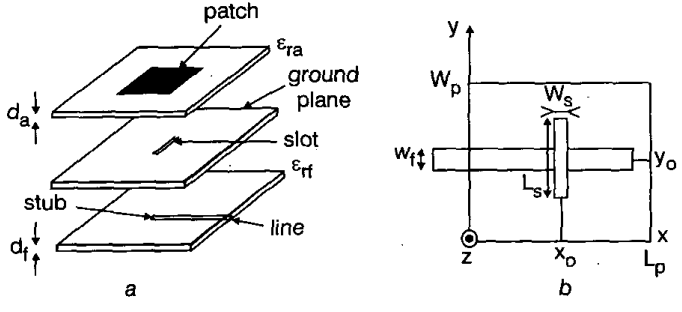

Fig. 1 Geometry of aperture coupled microstrip patch antenna with thick ground plane

$a$ View from side

$b$ View from top

Analysis: The cavity method [1] was used previously to describe the slot coupled patch antenna (Fig. 1) at low frequencies with very small slot thickness compared with the wavelength. In this case the input impedance is given by

$$
Z_{\text {in }}=\left(\frac{\Delta V}{V_{o}}\right)^{2} \frac{1}{Y_{\text {patch }}+Y_{\text {slot }}}+Z_{\text {stub }}
$$

where $Y_{\text {patch }}$ is the patch admittance given by the cavity method, $Y_{\text {slot }}$ is the slot admittance given by the transmission line model (the slot is considered as two parallel short circuited slot lines), $\Delta V / V_{o}$ is the transformation ratio corresponding to the modal voltage discontinuity of the slot in the microstrip feeding line, and $Z_{\text {stub }}$ represents the contribution of the open stub.

At millimetre wave frequencies, the ground plane, the thickness of which corresponds at the least to the copper thickness of the substrate, cannot be considered to be infinitely thin. In [2], the authors studied three methods to take into account the finite thickness of the ground plane in the cavity method (Fig. 4 in [2]).

When the thickness is $<0.025 \lambda_{o}$, method 3 seems to be the best with regard to Haddad's measurement. [3] and then an empirical correction factor $C_{f}$ is introduced in the cavity method. This coefficient is given by

$$
C_{f}=\frac{\exp \left\{\left(-2 \pi A t / \lambda_{c}\right) \sqrt{1-\left(\lambda_{c} / \lambda_{o}\right)^{2}}\right\}}{1-\left(\lambda_{c} / \lambda_{o}\right)^{2}}
$$

where

$$
\left\{\begin{array}{l}
\dot{\lambda}_{o}=3 \times 10^{8} / f \\
\dot{\lambda}_{c}=2 \cdot L_{s}
\end{array}\right.
$$

and $A$ is an experimental factor equal to 3 for a narrow rectangular slot. Then the input impedance becomes

$$
Z_{\text {in }}=\left(\frac{\Delta V}{V_{o}}\right)_{t=0}^{2} \frac{1}{Y_{\text {patch }}+Y_{\text {slot }}} \cdot C_{f}+Z_{\text {stub }}
$$

When the thickness becomes more important $\left(>0.025 \lambda_{0}\right)$, another method (method 2 in [2]) has been used. It considers an exponential attenuation to the power $2 \alpha_{10}$ for the $\mathrm{TE}_{10}$ mode in the slot. In this case, the input impedance is given by 